\title{
An Efficient Hybrid Hierarchical Agglomerative Clustering (HHAC) Technique for Partitioning Large Data Sets
}

\author{
P.A. Vijaya, M. Narasimha Murty, and D.K. Subramanian \\ Department of Computer Science and Automation, \\ Indian Institute of Science, \\ Bangalore - 560012, India \\ \{pav, mnm, dks\}@csa.iisc.ernet.in
}

\begin{abstract}
In this paper, an efficient Hybrid Hierarchical Agglomerative Clustering (HHAC) technique is proposed for effective clustering and prototype selection for pattern classification. It uses the characteristics of both partitional (an incremental scheme) and Hierarchical Agglomerative Clustering (HAC) schemes. Initially, an incremental, partitional clustering algorithm - leader is used for finding the subgroups/subclusters. It reduces the time and space requirements incurred in the formation of the subclusters using the conventional hierarchical agglomerative schemes or other methods. Further, only the subcluster representatives are merged to get a required number of clusters using a hierarchical agglomerative scheme which now requires less space and time when compared to that of using it on the entire training set. Thus, this hybrid scheme would be suitable for clustering large data sets and we can get a hierarchical structure consisting of clusters and subclusters. The subcluster representatives of a cluster can also handle its arbitrary/non-spherical shape. The experimental results (Classification Accuracy (CA) using the prototypes obtained and the computation time) of the proposed algorithm are promising.
\end{abstract}

\section{Introduction}

Clustering is basically an unsupervised learning technique to divide the data into groups of similar objects by using a distance or similarity function. Clustering is mainly used for prototype selection/abstractions for pattern classification, data reorganization and indexing and for detecting outliers and noisy patterns. The earlier approaches do not adequately consider the fact that the data set can be too large and may not fit in the main memory of some computers. It is necessary to examine the principle of clustering to devise efficient algorithms to minimize the I/O operations and space requirements and to get appropriate prototypes/abstractions to increase the Classification Accuracy (CA). Clustering techniques are classified into hierarchical and partitional methods [3,5]. Hierarchical clustering algorithms can be either divisive (top-down) or agglomerative (bottom-up). Single Link Algorithm (SLA) and Complete Link Algorithm 
(CLA) are Hierarchical Agglomerative Clustering (HAC) schemes which require the distance/proximity matrix [3].

$\mathrm{K}$-means, K-medoids and K-medians algorithms [3, 5] are partitional clustering approaches which are based on $K$ centroids, medoids and median patterns of the initial partitions respectively and are iteratively improved. In data mining applications, both the number of patterns and features are typically large. SLA, CLA, K-means, K-medoids and K-medians based algorithms are not feasible for large data sets. Following are some of the clustering approaches that have been used on large data sets. CLARA, CLARANS and CURE [5] clustering schemes are designed to find clusters using random samples (subset of the original set). Divide and conquer scheme, a data partitioning technique, is used for disk resident data sets [3. Incremental clustering methods such as leader based algorithm [3, 6] and BIRCH [5] are more efficient for clustering large data sets as they involve very few database scans (less I/O operations). Leader is a simple partitional clustering technique suitable for any type of data set such as numerical, categorical and sequence whereas BIRCH is an incremental hierarchical agglomerative clustering technique and is suitable only for numerical data sets. In [6], we had proposed an incremental top-down hierarchical approach for clustering large data sets using nearest leader based algorithm [7.

The problem we have considered here is: Given a large data set, design and implement an efficient hybrid technique to find a good set of prototypes from meaningful partitions/groupings so as to improve the CA and reduce the computation time and space requirements. In the literature, a fuzzy hybrid hierarchical clustering method has been used by Arnaud et al. 11 using fuzzy c-means and a hierarchical agglomerative scheme. A hybrid hierarchical clustering scheme has been designed for protein data set by Harlow et al. 2] using Markov clustering and a hierarchical agglomerative scheme. A hybrid clustering scheme has been developed by Mark et al. 4] for clustering genes using PAM (Partitioning Around Medoids) [5] and merging operations. Fuzzy c-means, PAM and Markov clustering methods are computationally expensive for large data sets when compared to leader based method. Also, a set of leaders may form a better set of prototypes than that of random samples chosen as prototypes. This is evident from Figure 4, drawn based on the experimental results for one of the data sets (Pendigits) used. Leader based algorithm is suitable for preprocessing or at the first level of a hybrid clustering scheme for large data sets.

In this paper, we propose a hybrid clustering method which combines the characteristics of an incremental, partitional clustering algorithm - leader and a hierarchical agglomerative clustering scheme. This clustering technique is suitable for numerical, categorical and sequence data sets with suitable similarity measures. In this paper, we report the results for numerical data sets and we use Euclidean distance for characterizing dissimilarity between two patterns. This paper is organized as follows. Section 2 contains the details of the proposed method. Experimental results and discussions are presented in section 3 . Conclusions are provided in section 4 . 


\section{Proposed Method}

In the proposed Hybrid Hierarchical Agglomerative Clustering (HHAC) algorithm, there are two phases. In the first phase, leader based algorithm is used to generate subclusters (say $S$ representatives, $S<n$, where $n$ is the total number of training patterns) using a suitable threshold value. In the second phase, these $S$ representatives are merged using a hierarchical agglomerative scheme such as SLA or CLA to obtain say $K$ clusters and thus $K$ cluster representatives. In SLA, the two clusters with the smallest minimum pairwise distance are merged at every level whereas in CLA, the two clusters with the smallest maximum pairwise distance are merged.

In leader algorithm, the first pattern is selected as the leader of a cluster and each of the remaining patterns is either assigned to one of the existing clusters or may become leader of a new cluster based on the user defined threshold value. There are two ways of assigning the next pattern to a cluster [7]. In one scheme, the next pattern is compared with the leaders created in the order one by one. It is assigned to the leader under consideration if the distance is less than or equal to the threshold value and the remaining leaders are not considered further. Let us refer to this method as 'Ordered Leader No Update (OLNU)' as the cluster representative is not updated. In the other scheme, the next pattern is tested with all the existing leaders and is assigned to the nearest leader/cluster

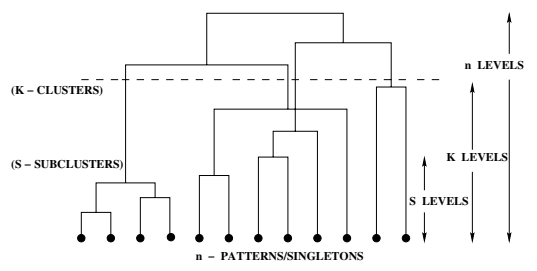

Fig. 1. Dendrogram of a HAC Algorithm

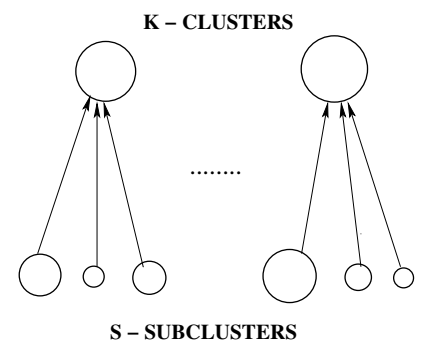

Fig. 3. Hierarchical Structure in HHAC Algorithm

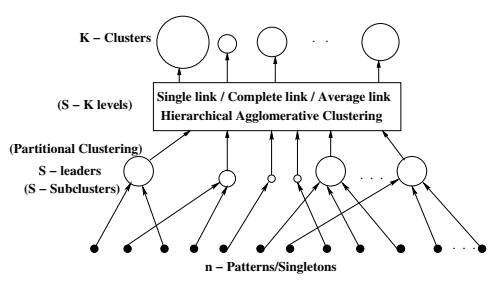

Fig. 2. Hybrid Hierarchical Agglomerative Clustering

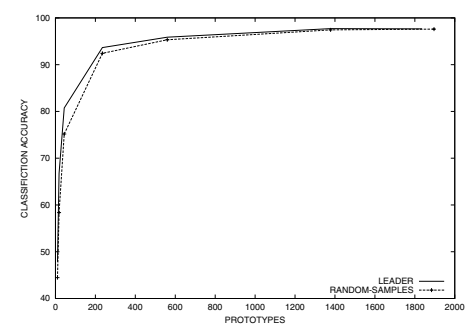

Fig. 4. Leader vs Random Samples 
if the distance is less than or equal to the threshold value. Let us refer to this method as 'Nearest Leader No Update (NLNU)'. OLNU/NLNU algorithm 77 requires only one database scan and its time complexity is $O(n S d)$, where $d$ is the total number of attributes in a pattern. OLNU method takes less training time than NLNU method 7 . We use OLNU method in the preclustering process or at the first stage in a bottom-up hybrid hierarchical clustering technique such as HHAC for large data sets when the number of subclusters formed is large.

A small threshold value is chosen (for a dissimilarity index measure) to obtain a set of subclusters so that its size is larger than the number of clusters to be generated. In HAC schemes, the subclusters are formed in the beginning and these steps are replaced by the incremental partitional scheme - leader. This is used to save the operations performed in the first $S$ levels in any HAC schemes as shown in Figure 1. These $S$ representatives are merged using a HAC scheme to obtain say $K$ clusters as shown in Figure 2. We use centroid as the cluster representative for numerical data at the second level.

Time and space complexity of the proposed method is less compared to the conventional HAC algorithms which use all the $n$ training patterns as singleton clusters. As $S<n, S$ representatives and $S \times S$ distance matrix may be accommodated in the main memory and hence reduces the disk I/O operations for large data sets. The space requirement of the HAC scheme in the second phase of HHAC is $O\left(S^{2}\right)$ instead of $O\left(n^{2}\right)$. Time complexity of the hierarchical agglomerative scheme is $O\left(S^{2} d\right)$ instead of $O\left(n^{2} d\right)$ for SLA and is $O\left(\left(S^{2} \log S\right) d\right)$ instead of $O\left(\left(n^{2} \log n\right) d\right)$ for CLA. Thus, HHAC algorithm generates a hierarchical structure as shown in Figure 3 and it consists of subgroups/subclusters within each cluster as required in many applications. Its time complexity is $O\left(\left(n S+S^{2}\right) d\right)$ or $O\left(\left(n S+S^{2} \log S\right) d\right)$ for leader with SLA or CLA respectively. There would be several subgroups/subclusters in each group/cluster of numerical data sets such as handwritten or printed character data sets. It is necessary to find these subgroups/subclusters also. Subcluster representatives of a cluster can take care of the arbitrary shape of that cluster. If a representative from each subgroup is chosen then naturally CA would be improved. Prototypes (representatives of the clusters and subclusters) are generated using the training data set. During classification/testing phase, for every test pattern, the nearest cluster representative is found first and then the nearest subcluster representative in that cluster is determined. Then the test pattern is classified based on the nearest of these two. For different values of threshold and $K$, experiments (both training and testing) are conducted and the results are evaluated. To evaluate the clustering quality (quality of the prototypes selected), labelled patterns are considered. During training phase they are treated as unlabelled patterns and the prototypes are selected. The quality of the prototypes is evaluated using the $\mathrm{CA}$ obtained on the test/validation data set. The experimental results of the proposed method are discussed in the following section. 


\section{$3 \quad$ Experimental Results}

To evaluate the performance of HHAC algorithm, experiments were conducted on many data sets from machine learning repository of University of California, Irvine (http://www.ics.uci.edu/). Because of the space constraints, we are reporting few results for one of the data sets - Pendigits. There are 7494 patterns in the training set, 3498 patterns in the testing set and 10 classes with 16 numerical attribute values.

Table 1. Time and Classification Accuracy (CA)

\begin{tabular}{lllllll}
\hline $\begin{array}{l}\text { Algo- } \\
\text { rithm }\end{array}$ & $\begin{array}{l}\text { Thres- } \\
\text { hold }\end{array}$ & $\begin{array}{l}\text { Subclusters } \\
(S)\end{array}$ & $\begin{array}{l}\text { Clusters } \\
(K)\end{array}$ & $\begin{array}{l}\text { Training } \\
\text { time }(\mathrm{s})\end{array}$ & $\begin{array}{l}\text { Testing } \\
\text { time }(\mathrm{s})\end{array}$ & $\begin{array}{l}\text { CA } \\
(\%)\end{array}$ \\
\hline NNC & - & - & 7494 & - & 135.57 & 97.74 \\
\hline SLA & - & - & 861 & 61662.97 & 14.98 & 87.19 \\
& - & - & 2298 & 55018.08 & 40.73 & 96.65 \\
\hline CLA & - & - & 861 & 68862.83 & 15.27 & 97.28 \\
& - & - & 2298 & 60241.55 & 40.76 & 97.88 \\
\hline LSLA(K) & 20 & 4816 & 861 & 15380.90 & 15.89 & 86.62 \\
& 20 & 4816 & 2298 & 11977.26 & 41.58 & 96.45 \\
& 25 & 3361 & 861 & 4799.22 & 13.37 & 91.19 \\
& 25 & 3361 & 2298 & 2760.94 & 41.60 & 97.36 \\
\hline LCLA(K) & 20 & 4816 & 861 & 16884.30 & 15.34 & 96.48 \\
& 20 & 4816 & 2298 & 12780.51 & 41.27 & 98.02 \\
& 25 & 3361 & 861 & 5021.54 & 13.08 & 95.91 \\
& 25 & 3361 & 2298 & 2839.51 & 40.16 & 97.51 \\
\hline LSLA(S+K) & 20 & 4816 & 861 & 15380.90 & 18.35 & 88.67 \\
& 20 & 4816 & 2298 & 11977.26 & 46.10 & 96.51 \\
& 25 & 3361 & 861 & 4799.22 & 16.93 & 92.45 \\
& 25 & 3361 & 2298 & 2760.94 & 41.99 & 97.48 \\
\hline LCLA(S+K) & 20 & 4816 & 861 & 16884.30 & 18.27 & 97.25 \\
& 20 & 4816 & 2298 & 12780.51 & 42.48 & 98.02 \\
& 25 & 3361 & 861 & 5021.54 & 14.33 & 97.02 \\
& 25 & 3361 & 2298 & 2839.51 & 40.55 & 97.62 \\
\hline
\end{tabular}

LSLA - Leader and SLA, LCLA - Leader and CLA. K or S+K within brackets indicates the set of prototypes used during testing phase

The experiments were done on an Intel pentium- 4 processor based machine having a clock frequency of $1700 \mathrm{Mhz}$ and $512 \mathrm{MB}$ RAM. In each case, different threshold and $K$ values were used for HHAC algorithms. The performances of Nearest Neighbour Classifier (NNC) [3, 6] and HAC schemes have also been reported in Table 1. Performance with CLA is the best among the four HAC schemes - SLA, CLA, Average Link algorithm and Weighted Average Link algorithm, for the data sets used. We have reported the results for SLA and CLA. 
From the results obtained (as shown in Table 1), it is evident that HHAC algorithms performed well compared to HAC approaches in terms of computation time for almost the same CA or even more. Training time is the time taken for generating the prototypes. Testing time is the time taken for classifying all the test patterns. After the training phase, testing time is less when only cluster representatives are used but increases a little when subcluster representatives are also used. Even then, it is less when compared to NNC. The CA is greater than or as that of NNC when both cluster and subcluster representatives are used. Even if more number of prototypes are generated, classification time is less as only a part of the hierarchical structure is searched.

\section{Conclusions}

In this paper, experimental results of HHAC algorithm on numerical data sets show that it performs well by properly tuning the threshold and $K$ values. HHAC algorithm is used to generate clusters and subclusters - a hierarchical structure. If both cluster and subcluster representatives are used then the CA is nearly same as that of NNC or even more. Subclusters can be obtained at low computation cost when compared to the conventional HAC schemes or other partitional methods, by using an incremental partitional approach such as leader. The performance of the hybrid technique with K-means or K-medoids at the second level is also found to be good. The proposed technique can also be used on protein sequence data sets, text and web document collection with appropriate distance measures.

\section{References}

1. Arnaud, D., Patrice, B., Gerard, V. L.: A Fuzzy Hybrid Hierarchical Clustering Method with a New Criterionable to Find the Optimal Partition. Fuzzy Sets and Systems. 128:3(2002) 323-338

2. Harlow, T. J., Gogarten, J. P., Ragan, M. A.: A Hybrid Clustering Approach to Recognition of Protein Families in 114 Microbial Genomes. BMC Bioinformatics. 5:45(2004) 1-14

3. Jain, A. K., Murty, M. N., Flynn, P. J.: Data Clustering: A Review. ACM Computing Surveys. 31:3(1999) 264-323

4. Mark, J. V., Katherine S. P.: A New Algorithm for Hybrid Hierarchical Clustering with Visualization and the Bootstrap. Journal of Statistical Planning and Inference. 117:2(2003) 275-303

5. Pujari, A. K.: Data Mining Techniques. Universities Press (India) Private Limited. (2000)

6. Vijaya, P. A., Murty, M. N., Subramanian, D. K.: Leaders-Subleaders: An Efficient Hierarchical Clustering Algorithm for Large Data Sets. Pattern Recognition Letters. 25(2004) 503-511

7. Vijaya, P. A., Murty, M. N., Subramanian, D. K.: Analysis of Leader based Clustering Algorithms for Pattern Classification, Accepted for publication in the Proceedings of the $2^{\text {nd }}$ IICAI, Pune, India, Dec. 2005. 